\title{
Low-fat dairy products and blood pressure: follow-up of 2290 older persons at high cardiovascular risk participating in the PREDIMED study
}

\author{
Estefanía Toledo ${ }^{1}$, Miguel Delgado-Rodríguez ${ }^{2}$, Ramón Estruch $^{3,4}$, Jordi Salas-Salvadó ${ }^{4,5}$,
} Dolores Corella $^{4,6}$, Enrique Gomez-Gracia ${ }^{7}$, Miquel Fiol ${ }^{4,8}$, Rosa M. Lamuela-Raventós ${ }^{9}$, Helmut Schröder ${ }^{4,10}$, Fernando Arós ${ }^{11}$, Emilio Ros ${ }^{4,12}$, Valentina Ruíz-Gutiérrez ${ }^{13}$, Jose Lapetra ${ }^{4,14}$, Manuel Conde-Herrera ${ }^{15}$, Guillermo Sáez ${ }^{16}$, Ernest Vinyoles ${ }^{4,17}$ and Miguel A. Martínez-González ${ }^{1 *}$

${ }^{1}$ Department of Preventive Medicine and Public Health, School of Medicine - Clinica Universitaria de Navarra, University of Navarra, Irunlarrea 1, 31080 Pamplona, Navarra, Spain

${ }^{2}$ Division of Preventive Medicine and Public Health, University of Jaen, Paraje Lagunillas, 23071 Jaen, Spain

${ }^{3}$ Department of Internal Medicine, Hospital Clinic, Villarroel 170, 08036 Barcelona, Spain

${ }^{4}$ CIBER Fisiopatología Obesidad y Nutrición (CB06/03), Instituto de Salud Carlos III, Spain

${ }^{5}$ Human Nutrition Department, School of Medicine, University Rovira i Virgili, San Llorenc 21, 43201 Reus, Tarragona, Spain ${ }^{6}$ Department of Preventive Medicine, School of Medicine, University of Valencia, Avda. Blasco Ibanez 15, 46010 Valencia, Spain ${ }^{7}$ Department of Epidemiology, School of Medicine, University of Malaga, Campus de Teatinos s/n, 29071 Malaga, Spain

${ }^{8}$ Department of Cardiology, Hospital Universitario Son Dureta, Andrea Doria 55, 07014 Palma de Mallorca, Spain

${ }^{9}$ Department of Nutrition and Bromatology, School of Pharmacy, Avda. Joan XXIII s/n, Barcelona, Spain

${ }^{10}$ Lipids and Cardiovascular Epidemiology Research Unit, Municipal Institute for Medical Research (IMIM), Carrer Doctor

Aiguader 80, 08003 Barcelona, Spain

${ }^{11}$ Department of Cardiology, Hospital Txagorritxu, Jose Achotegui s/n, 01009 Vitoria, Alava, Spain

${ }^{12}$ Lipid Clinic, Endocrinology and Nutrition Service, Hospital Clinic, Villarroel 170, 08036 Barcelona, Spain

${ }^{13}$ Instituto de la Grasa, Consejo Superior de Investigaciones Científicas (CSIC), Avda. Padre Garcia Tejero 4, 41012 Sevilla, Spain

${ }^{14}$ Department of Family Medicine, Primary Care Division of Sevilla, San Pablo Health Center, Damasco s/n, 41007 Sevilla, Spain

${ }^{15}$ Department of Epidemiology and Public Health, Hospital Virgen del Rocío, Avda. Manuel Siurot s/n, 41013 Sevilla, Spain

${ }^{16}$ Department of Biochemistry, School of Medicine, University of Valencia, Avda. Blasco Ibánez 15, 46010 Valencia, Spain

${ }^{17}$ Institut Catalá de la Salut (ICS), Avda. Gran Vía de las Corts Catalanes 587, Atico, Barcelona, Spain

(Received 3 January 2008 - Revised 13 March 2008 - Accepted 30 March 2008 - First published online 20 May 2008)

High blood pressure (BP) has been ranked as the most important risk factor worldwide regarding attributable deaths. Dietary habits are major determinants of BP. Among them, frequent intake of low-fat dairy products may protect against hypertension. Our aim was to assess the relationship between low-fat dairy product intake and BP levels and their changes after 12-month follow-up in a cohort of asymptomatic older persons at high cardiovascular risk recruited into a large-scale trial assessing the effects of Mediterranean diets on cardiovascular outcomes. Data from 2290 participants, including 1845 with hypertension, were available for analyses. Dairy products were not a specific part of the intervention; thus, data were analysed as an observational cohort. Dietary information was collected with validated semi-quantitative FFQ and trained personnel measured BP. To assess BP changes, we undertook cross-sectional analyses at baseline and at the end of follow-up and longitudinal analyses. A statistically significant inverse association between low-fat dairy product intake and systolic BP was observed for the 12-month longitudinal analysis. In the longitudinal analysis, the adjusted systolic and diastolic BP were significantly lower in the highest quintile of low-fat dairy product intake $(-4.2(95 \% \mathrm{CI}-6.9,-1.4)$ and $-1.8(95 \%$ $\mathrm{CI}-3 \cdot 2,-0 \cdot 4) \mathrm{mmHg}$ respectively), whereas the point estimates for the difference in diastolic BP indicated a modest non-significant inverse association. Intake of low-fat dairy products was inversely associated with BP in an older population at high cardiovascular risk, suggesting a possible protective effect against hypertension.

Low-fat dairy products: Blood pressure: Dietary pattern: Cardiovascular risk: PREDIMED study

Worldwide, it has been estimated that there are one billion hypertensive individuals ${ }^{(1)}$ and that hypertension is the most important attributable risk factor for death ${ }^{(2)}$. Small population reductions in blood pressure $(\mathrm{BP})$ of $2-5 \mathrm{mmHg}$ are translated into important reductions in mortality due to stroke and CHD and in total mortality ${ }^{(3)}$. For this reason, new approaches

Abbreviations: BP, blood pressure; DBP, diastolic BP; SBP, systolic BP.

* Corresponding author: Professor Miguel A. Martínez-González, fax +34948 425649, email mamartinez@unav.es 
should be evaluated and implemented to reduce BP levels. Although diet is acknowledged as a major determinant of BP, the role of some foods is still controversial. One preventive strategy that has been shown to be beneficial is the DASH diet ${ }^{(4)}$. The results from the DASH trial suggest that a dietary pattern rich in fruits and vegetables, poor in SFA and with increased intake of low-fat dairy products can be an effective means to prevent the development of hypertension. The DASH trial tested the effects on BP of a complex dietary pattern, not of particular foods (i.e. low-fat dairy products); thus, there is no clinical trial evidence to support a specific BP-lowering effect of low-fat dairy products. Nonetheless, the results of a large prospective observational cohort, the SUN Study ${ }^{(5)}$, suggested that the intake of lowfat dairy products was associated with a reduction of incident hypertension. Indeed, participants in the highest quintile of low-fat dairy intake halved their risk of developing hypertension. In the CARDIA study, also a prospective study, the authors found an inverse association between total dairy product intake and 10-year cumulative incidence of hypertension among overweight people ${ }^{(6)}$. These results were partially confirmed by those of the National Heart, Lung, and Blood Institute Family Heart Study ${ }^{(7)}$, in which an inverse association between consumption of dairy products and prevalence of hypertension was observed. However, this was a cross-sectional study that did not assess the specific relationship of low-fat dairy products to BP.

From a biological point of view, protection against hypertension by low-fat dairy products has been attributed to their nutrient composition. Dairy products are good sources of $\mathrm{K}$, $\mathrm{Mg}, \mathrm{Ca}$, peptides with an inhibitory effect on angiotensin-Iconverting enzyme activity and opioid-like peptides ${ }^{(8,9,10)}$. On the other hand, whole-fat dairy products are rich in SFA, and high-SFA diets impair endothelial function ${ }^{(11)}$. All these mechanisms add biological plausibility to the hypothesis of a specific protection against hypertension provided by lowfat dairy products. There is scarce epidemiological evidence of a specific effect on BP of these foods in persons at elevated cardiovascular risk with a high frequency of treated hypertension. The aim of the present study was to assess the relationship between low-fat dairy product intake and BP in an observational follow-up study conducted in a large population at high cardiovascular risk participating in the PREDIMED study, a trial to test the effects of the Mediterranean diet on cardiovascular outcomes ${ }^{(12)}$.

\section{Methods}

\section{Study population}

The present study has been conducted within the frame of the PREDIMED trial. The design of the PREDIMED trial has been reported in detail elsewhere ${ }^{(12)}$. Briefly, the PREDIMED trial is a large, parallel-group, multicentre, randomized and controlled 4-year clinical trial that aims to assess the effects of a Mediterranean-type diet on CVD and in which participants are assigned to one of three different dietary patterns (low-fat diet; Mediterranean diet supplemented with nuts; Mediterranean diet supplemented with olive oil) ${ }^{(12)}$. The study population is composed of men aged between 55 and 80 years and women aged between 60 and 80 years with no previously documented CVD but at high cardiovascular risk. Inclusion criteria were either diabetes mellitus type 2 or at least three of the following risk factors: current smoking; hypertension; hyperlipidaemia; HDL-cholesterol $<1.034 \mathrm{mmol} / \mathrm{L}$; overweight/obesity; family history of premature CHD.

In the present analysis, data from this trial were analysed as an observational cohort study because the evaluated exposure (intake of low-fat dairy products) was not a part of the intervention.

In the present analyses we included data from the first enrolled participants in the PREDIMED trial, in which the retention rate during the first year was greater than $90 \%$. These earlier participants were not significantly different regarding socio-demographic characteristics and risk factors from those subsequently recruited for the trial. Data from 2392 participants who completed questionnaires at baseline and after 12 months of follow-up were available. We excluded sixteen participants who had missing values for baseline BP and twenty-seven with missing BP data at 12 months. Participants reporting extreme total energy intakes with values outside pre-specified limits (2093 to $14650 \mathrm{~kJ} / \mathrm{d}$ in women and 3349 to $16743 \mathrm{~kJ} / \mathrm{d}$ in men $)^{(13)}$ were excluded from the analysis. The effective sample size for statistical analyses was 2290. All participants provided informed consent and the protocol was approved by the institutional review boards of all participating centres according to the Declaration of Helsinki ${ }^{(14)}$.

\section{Dietary assessment}

The dietary habits of participants, both at baseline and after follow-up for 12 months, were assessed using a semi-quantitative 137 -item FFQ previously validated in Spain ${ }^{(15)}$. The questionnaire was based on the typical portion sizes that were multiplied by the consumption frequency for each food. Nutrient composition was estimated from Spanish food composition tables ${ }^{(16,17)}$.

Information about dairy products was assessed in fifteen items of the semi-quantitative FFQ (whole-fat milk, partially skimmed milk, skimmed milk, condensed milk, whipped cream, yoghurt, skimmed yoghurt, milkshake, ricotta cheese or junket, petit Suisse cheese, spreadable cheese wedges, cottage cheese, other cheese, custard, and ice cream). Partially skimmed milk, skimmed milk, skimmed yoghurt and cottage cheese were considered low-fat dairy products.

\section{Blood pressure measurements}

At baseline and after 12 months follow-up, trained personnel measured $\mathrm{BP}$ in triplicate with a validated semi-automatic oscillometer (Omron HEM-705CP; Hoofddorp, The Netherlands) while the participant was in a seated position after 5 min rest. The mean of the three systolic BP (SBP) and diastolic BP (DBP) measurements was calculated ${ }^{(18,19)}$. Arm circumference determined the cuff size and BP was measured in the forearm at heart level.

\section{Assessment of other covariates}

Smoking status was categorized into never, current or past smoking according to self-reports. Participants were considered as diabetic, hyperlipidaemic or hypertensive if they had a previous diagnosis of these conditions and/or they were treated with anti-diabetic, cholesterol-lowering or anti-hypertensive 
agents, respectively. Information about treatment with non-steroidal anti-inflammatory drugs, drugs that may decrease or increase serum $\mathrm{Ca}$ levels or $\mathrm{K}$ supplements was also collected.

The highest achieved educational level was recorded. Physical activity was determined with the validated Spanish version of the Minnesota questionnaire ${ }^{(20,21)}$. The mean year metabolic rate was derived from the information on sixty-seven different activities and their energy expenditure and expressed in $\mathrm{kJ} / \mathrm{d}$. Trained personnel measured height and weight with calibrated scales and wall-mounted stadiometers, respectively. BMI was calculated as the weight divided by the square of the height $\left(\mathrm{kg} / \mathrm{m}^{2}\right)$.

At each visit, information on medication intake of each participant was collected for oral anti-diabetic agents and insulin treatment, cholesterol-lowering drugs (statins, fibrates, resins or others) and anti-hypertensive agents (angiotensin-converting enzyme inhibitors, inhibitors of the angiotensin II AT1 receptors, other anti-hypertensive drugs (anti-adrenergics with peripheral action, arteriolar vasodilators (including $\mathrm{Ca}$ entry blockers), diuretics and nitrates)). Information about treatment with non-steroidal anti-inflammatory drugs, drugs that may decrease or increase serum $\mathrm{Ca}$ levels or $\mathrm{K}$ supplements was also collected.

\section{Statistical analysis}

Food and nutrient intakes were adjusted for total energy intake using the residual method ${ }^{(13)}$. Missing values in potential confounding factors were entered as an additional category in the multivariable models.

Participants were divided into five groups according to quintiles of consumption of low-fat dairy or whole-fat dairy products. Hereby, we avoided making specific assumptions about the shape of the dose-response relationship and thus minimized the influence of outliers ${ }^{(13)}$. First, we compared the crude means for SBP and DBP across quintiles of dairy product consumption (separately for low-fat and whole-fat items). Afterwards, multivariable linear regression models were fit to control for potential confounders, such as the presence of diabetes mellitus, mean physical activity during the previous year, anti-hypertensive treatment, treatment with non-steroidal anti-inflammatory drugs or drugs that may modify serum $\mathrm{Ca}$, alcohol consumption, use of $\mathrm{K}$ supplements, total energy intake, intake of $\mathrm{Na}$ (including $\mathrm{Na}$ from salt used for seasoning meals), of SFA, MUFA and fibre, consumption of fruits and vegetables and the non-dairy intake of $\mathrm{K}, \mathrm{Ca}, \mathrm{Mg}$ and total protein. Fish and whole grain intakes were also considered as potentially confounding factors in ancillary analyses. Multivariable linear regression models were fitted considering current BMI as a potential confounder. However, current BMI may also be considered as an intermediate variable in the association between low-fat dairy consumption and BP and, therefore, we constructed models both adjusted for BMI and not adjusted for BMI. Although smoking status and hyperlipidaemia have not been established as risk factors for hypertension, they were also controlled for as indicators of lifestyle-related factors.

For each exposure (low-fat and whole-fat dairy products) we fitted five multivariable linear regression models: (1) a baseline cross-sectional analysis (exposure: baseline dairy product consumption; outcome: baseline BP); (2) a 12-month cross-sectional analysis (exposure: dairy product consumption after 12 months; outcome: BP after 12 months); (3) a conventional longitudinal analysis (exposure: baseline dairy product consumption; outcome: BP after 12 months); (4) a longitudinal analysis with BP changes as outcome (exposure: baseline dairy product consumption; outcome: BP changes during follow-up); (5) a dynamic longitudinal analysis (exposure: changes in dairy product consumption; outcome: changes in $\mathrm{BP})$. In the models in which the outcome was 12 months BP, $\mathrm{BP}$ changes and/or when the exposure was 12 months dairy product consumption, we additionally adjusted for the intervention group. Our sample size provided a statistical power greater than $90 \%$ to detect between-quintile (first $v$. fifth) differences of 3 and $2 \mathrm{mmHg}$ in SBP and DBP changes, respectively, assuming standard deviation values of $13 \mathrm{mmHg}$ for SBP and 7 for DBP. For linear trend tests, each quintile of dairy consumption was assigned its median value and the resulting variable was considered as quantitative in the multivariable analyses. We tested interactions between low-fat or whole-fat dairy products consumption and obesity, diabetes mellitus, hypertension and treatment with anti-hypertensive agents in the conventional longitudinal analyses. CI at $95 \%$ were calculated in every model including all the data.

All significance levels were calculated two-sided and all analyses were performed with SPSS version 13.0 (SPSS Inc., Chicago, IL, USA).

\section{Results}

The characteristics of participants are presented in Table 1 according to their baseline consumption of low-fat dairy products. Women and never-smokers consumed more and past smokers consumed less low-fat dairy products. Participants with a higher consumption of low-fat dairy products also had a lower intake of total energy, alcohol, $\mathrm{Ca}$ and protein from non-low-fat dairy sources. Low-fat and whole-fat dairy consumption were inversely correlated $(r-0.43 ; P<0.001)$.

Tables 2 and 3 show the BP levels and their changes during follow-up according to low-fat and whole-fat dairy products consumption, respectively.

Regarding low-fat dairy consumption, we found inverse linear trends for SBP that were statistically significant in the cross-sectional analysis after the 12 months follow-up. The test of linear trend in the cross-sectional analyses at baseline was also marginally significant and the CI did not include the null value for the comparison of SBP levels between the upper and lower quintiles of low-fat dairy products in both cross-sectional analyses. More importantly, in the conventional longitudinal analysis with SBP levels after 12 months, we found an inverse linear trend and the CI for the comparison of SBP across extreme quintiles of low-fat dairy product consumption did not include the null value. When we did not adjust the multivariable regression model for current BMI, the comparison between extreme quintiles and the linear trend did not appreciably change (data not shown). All point estimates for the comparison between extreme quintiles showed a negative sign, indicating an inverse association between low-fat dairy product consumption and SBP. Only one of the adjusted analyses for DBP suggested an inverse association for the consumption of low-fat dairy products ( $P$ for trend 0.01 for the cross-sectional 
Table 1. Baseline characteristics of 2290 participants in the PREDIMED trial across quintiles of low-fat dairy consumption (Means and standard deviations or percentages)

\begin{tabular}{|c|c|c|c|c|c|c|c|c|c|c|c|}
\hline \multirow{3}{*}{ Sample size $(n) \ldots$} & \multicolumn{10}{|c|}{ Quintiles of low-fat dairy consumption at baseline } & \multirow[b]{3}{*}{$P$ for trend } \\
\hline & \multicolumn{2}{|c|}{$\begin{array}{c}\text { Q1 } \\
458\end{array}$} & \multicolumn{2}{|c|}{$\begin{array}{c}\text { Q2 } \\
458\end{array}$} & \multicolumn{2}{|c|}{$\begin{array}{c}\text { Q3 } \\
458\end{array}$} & \multicolumn{2}{|c|}{$\begin{array}{c}\text { Q4 } \\
458\end{array}$} & \multicolumn{2}{|c|}{$\begin{array}{c}\text { Q5 } \\
458\end{array}$} & \\
\hline & Mean & SD & Mean & SD & Mean & SD & Mean & SD & Mean & SD & \\
\hline Low-fat dairy products (g/d) & 3.1 & $17 \cdot 1$ & $141 \cdot 1$ & $53 \cdot 3$ & $236 \cdot 7$ & 26.8 & $406 \cdot 1$ & $80 \cdot 1$ & 631.6 & 139.4 & \\
\hline Total dairy products $(\mathrm{g} / \mathrm{d})$ & $262 \cdot 1$ & $216 \cdot 0$ & 238.0 & $121 \cdot 4$ & 295.4 & 67.6 & 460.9 & 119.9 & 664.9 & $146 \cdot 1$ & \\
\hline Whole-fat dairy products $(\mathrm{g} / \mathrm{d})$ & $261 \cdot 1$ & 218.3 & 99.0 & $123 \cdot 3$ & 60.6 & 63.9 & 56.8 & 84.2 & 34.9 & 55.9 & \\
\hline Age (years) $\dagger$ & $67 \cdot 2$ & 6.4 & $66 \cdot 7$ & $6 \cdot 1$ & $67 \cdot 0$ & $6 \cdot 1$ & 67.7 & 5.9 & $68 \cdot 1$ & 5.9 & 0.001 \\
\hline Sex (\% females) & \multicolumn{2}{|c|}{37.3} & \multicolumn{2}{|c|}{44.3} & \multicolumn{2}{|c|}{55.4} & \multicolumn{2}{|c|}{64.9} & \multicolumn{2}{|c|}{73.8} & $<0.001$ \\
\hline $\mathrm{BMI}\left(\mathrm{kg} / \mathrm{m}^{2}\right) \ddagger$ & 29.6 & 3.5 & 29.5 & 3.5 & 29.9 & 3.6 & 29.7 & 3.7 & 30.0 & 4.3 & 0.04 \\
\hline Educational level (\%)§ & \multirow{2}{*}{\multicolumn{2}{|c|}{$25 \cdot 8$}} & \multirow{2}{*}{\multicolumn{2}{|c|}{26.4}} & \multirow{2}{*}{\multicolumn{2}{|c|}{$26 \cdot 4$}} & \multirow{2}{*}{\multicolumn{2}{|c|}{$21 \cdot 8$}} & \multirow{2}{*}{\multicolumn{2}{|c|}{$21 \cdot 0$}} & \\
\hline$>$ secondary & & & & & & & & & & & 0.03 \\
\hline Physical activity (kJ/d) & 1102 & 1018 & 1109 & 1015 & 1073 & 957 & 1018 & 929 & 1049 & 956 & 0.17 \\
\hline Smokers (\%) & & & & & & & & & & & \\
\hline Past & \multicolumn{2}{|c|}{$32 \cdot 3$} & \multicolumn{2}{|c|}{28.4} & \multicolumn{2}{|c|}{$26 \cdot 6$} & \multicolumn{2}{|c|}{$19 \cdot 0$} & \multicolumn{2}{|c|}{$17 \cdot 0$} & $<0.001$ \\
\hline Current & \multirow{2}{*}{\multicolumn{2}{|c|}{$\begin{array}{l}20 \cdot 1 \\
63 \cdot 1\end{array}$}} & \multirow{2}{*}{\multicolumn{2}{|c|}{15.9}} & & & & & & & $<0.001$ \\
\hline Hyperlipidaemia (\%)\| & & & & & & & & & & & 0.02 \\
\hline Diabetes mellitus (\%) & & & & & & & & & & & 0.23 \\
\hline Hypertension (\%) & & & & & & & & & & & 0.03 \\
\hline Use of NSAID $(\%)$ & & & & & & & & & & & 0.04 \\
\hline Use of ACE inhibitors or ARA (\%) & & & & & & & & & & & 0.03 \\
\hline Use of other anti-hypertensive drugs (\%) & & & & & & & & & & & 0.07 \\
\hline Use of drugs that may rise calcaemia (\%)ף & & & & & & & & & & & 0.07 \\
\hline Use of drugs that may lower calcaemia $(\%)^{\star *}$ & & & & & & & & & & & 0.05 \\
\hline K supplements (\%) & & & & & & & & & & & 0.54 \\
\hline Total energy intake $(\mathrm{kJ} / \mathrm{d})$ & 10208 & 2297 & 9460 & 2351 & 9230 & 2335 & 9314 & 2059 & 9335 & 2100 & $<0.001$ \\
\hline $\mathrm{Na}(\mathrm{mg} / \mathrm{d})$ & 2366 & 696 & 2374 & 582 & 2355 & 542 & 2349 & 611 & 2333 & 571 & 0.30 \\
\hline $\mathrm{K}$ from other sources than dairy $(\mathrm{mg} / \mathrm{d})$ & 4026 & 805 & 3881 & 741 & 3863 & 756 & 3755 & 721 & 3685 & 802 & $<0.001$ \\
\hline Ca from other sources than dairy products $(\mathrm{mg} / \mathrm{d})$ & 837 & 300 & 640 & 201 & 585 & 171 & 575 & 197 & 523 & 173 & $<0.001$ \\
\hline $\mathrm{Mg}$ from other sources than dairy products $(\mathrm{mg} / \mathrm{d})$ & 352 & 71 & 342 & 66 & 337 & 65 & 324 & 63 & 316 & 6689 & $<0.001$ \\
\hline Alcohol $(\mathrm{g} / \mathrm{d})$ & 21.5 & 55.4 & 34.1 & 128.5 & 19.7 & $48 \cdot 3$ & 11.3 & 31.5 & 8.5 & $27 \cdot 1$ & $<0.001$ \\
\hline Fat (\% total energy intake) & 40.5 & 6.7 & 40.2 & 6.6 & 39.3 & 6.9 & 39.6 & 6.4 & 38.5 & $7 \cdot 0$ & $<0.001$ \\
\hline SFA (\% total energy intake) & 10.7 & $2 \cdot 3$ & 9.9 & $2 \cdot 2$ & 9.7 & $2 \cdot 1$ & 9.9 & $2 \cdot 2$ & 9.7 & $2 \cdot 2$ & $<0.001$ \\
\hline MUFA (\% total energy intake) & $20 \cdot 2$ & 4.5 & $20 \cdot 2$ & 4.4 & 19.9 & 4.5 & 20.1 & $4 \cdot 3$ & 19.3 & 4.7 & 0.003 \\
\hline Protein from other sources than dairy $(\mathrm{g} / \mathrm{d})$ & 87.0 & 13.5 & $82 \cdot 2$ & $12 \cdot 7$ & 81.7 & 12.7 & 78.3 & 12.9 & 74.4 & $13 \cdot 0$ & $<0.001$ \\
\hline Fibre $(g / d)$ & 24.0 & 7.9 & 24.8 & $7 \cdot 2$ & 24.9 & $7 \cdot 0$ & $24 \cdot 8$ & 7.0 & 24.7 & 7.4 & 0.26 \\
\hline Vegetables (g/d) & 308.7 & 151.5 & 317.9 & 129.9 & 321.1 & $138 \cdot 1$ & 322.3 & $130 \cdot 0$ & $325 \cdot 4$ & 159.7 & 0.07 \\
\hline Fruits $(\mathrm{g} / \mathrm{d})$ & $350 \cdot 1$ & $216 \cdot 6$ & $362 \cdot 0$ & 207.8 & 363.9 & $205 \cdot 1$ & 366.5 & $182 \cdot 3$ & 371.7 & 195.9 & 0.11 \\
\hline
\end{tabular}

NSAID, non-steroid anti-inflammatory drugs; ACE, angiotensin-converting enzyme; ARA, angiotensin II receptor antagonists. For details of subjects and procedures, see Methods.

Missing values: $\dagger 7 ; \ddagger 41 ; \S 64 ; \| 1$

I Use of Ca supplements and/or vitamin $D$.

*Use of bisphosphonates and/or calcitonin. 
Table 2. Blood pressure (BP) levels and BP changes across quintiles of low-fat dairy consumption* (Means and $95 \% \mathrm{Cl}$ )

\begin{tabular}{|c|c|c|c|c|c|c|c|c|c|c|c|c|}
\hline \multirow[b]{2}{*}{ Sample size... } & \multicolumn{6}{|c|}{ Systolic BP } & \multicolumn{6}{|c|}{ Diastolic BP } \\
\hline & 458 & $\begin{array}{l}\text { Q2 } \\
458\end{array}$ & 458 & $\begin{array}{l}\text { Q4 } \\
458\end{array}$ & $\begin{array}{l}\text { Q5 } \\
458\end{array}$ & $\begin{array}{l}P \text { for } \\
\text { trend }\end{array}$ & $\begin{array}{l}\text { Q1 } \\
458\end{array}$ & $\begin{array}{l}\text { Q2 } \\
458\end{array}$ & $\begin{array}{l}\text { Q3 } \\
458\end{array}$ & $\begin{array}{l}\text { Q4 } \\
458\end{array}$ & $\begin{array}{l}\text { Q5 } \\
458\end{array}$ & $\begin{array}{l}P \text { for } \\
\text { trend }\end{array}$ \\
\hline \multicolumn{13}{|c|}{ Baseline, cross-sectional } \\
\hline Mean BP & $152 \cdot 7$ & $150 \cdot 0$ & $150 \cdot 3$ & $150 \cdot 9$ & $148 \cdot 8$ & 0.01 & 84.9 & 83.5 & 83.6 & 84.2 & $82 \cdot 4$ & 0.003 \\
\hline $95 \% \mathrm{Cl}$ & $150 \cdot 8,154 \cdot 6$ & $147 \cdot 5,152 \cdot 5$ & $147 \cdot 8,152 \cdot 8$ & $148 \cdot 4,153 \cdot 4$ & $146 \cdot 2,151 \cdot 3$ & & $83 \cdot 8,85 \cdot 9$ & $82 \cdot 2,84 \cdot 9$ & $82 \cdot 2,84 \cdot 5$ & $82 \cdot 8,85 \cdot 5$ & $81 \cdot 0,83 \cdot 8$ & \\
\hline $\begin{array}{l}\text { Adjusted } \\
\text { model } \dagger\end{array}$ & 0 & $-2 \cdot 7$ & $-2 \cdot 9$ & -1.9 & -3.7 & 0.05 & 0 & -1.4 & -1.4 & -0.4 & $-1 \cdot 7$ & 0.13 \\
\hline $\begin{array}{l}95 \% \mathrm{Cl} \\
12 \text { month cross }\end{array}$ & ional ${ }^{(r e f)}$ & $-5 \cdot 2,-0 \cdot 1$ & $-5 \cdot 5,-0.3$ & $-4 \cdot 5,0 \cdot 8$ & $-6.5,-0.9$ & & (ref) & $-2 \cdot 7,-0.1$ & $-2 \cdot 8,-0.06$ & $-1 \cdot 8,1 \cdot 0$ & $-3.2,-0.3$ & \\
\hline Mean BP & 151.5 & $148 \cdot 7$ & $149 \cdot 4$ & $148 \cdot 2$ & $147 \cdot 6$ & 0.002 & 83.9 & 83.1 & $82 \cdot 0$ & $82 \cdot 1$ & $82 \cdot 3$ & 0.01 \\
\hline $95 \% \mathrm{Cl}$ & $149 \cdot 5,153 \cdot 4$ & $146 \cdot 2,151 \cdot 1$ & $146 \cdot 9,151 \cdot 8$ & $145 \cdot 8,150 \cdot 7$ & $145 \cdot 1,150 \cdot 0$ & & $82 \cdot 9,84.9$ & $81 \cdot 8,84 \cdot 5$ & $80 \cdot 7,83 \cdot 3$ & $80 \cdot 8,83 \cdot 4$ & $81 \cdot 0,83 \cdot 6$ & \\
\hline $\begin{array}{l}\text { Adjusted } \\
\text { model }\end{array}$ & 0 & $-3 \cdot 2$ & $-2 \cdot 2$ & -3.6 & -4.5 & 0.001 & 0 & -1.0 & $-1 \cdot 1$ & $-2 \cdot 2$ & -1.4 & 0.01 \\
\hline $\begin{array}{l}95 \% \mathrm{Cl} \\
\text { Longitudinal, ba } \\
\text { exposure } 12\end{array}$ & $e^{\text {(ref) }}$ & $-5 \cdot 7,-0.8$ & $-4 \cdot 7,0.3$ & $-6 \cdot 1,-1 \cdot 1$ & $-7 \cdot 1,-2 \cdot 0$ & & (ref) & $-2 \cdot 3,0 \cdot 2$ & $-2 \cdot 4,0.2$ & $-3 \cdot 5,-1 \cdot 0$ & $-2.7,-0.1$ & \\
\hline Mean BP & $150 \cdot 9$ & $148 \cdot 6$ & $149 \cdot 2$ & $149 \cdot 3$ & $147 \cdot 3$ & 0.02 & 83.4 & $82 \cdot 4$ & $82 \cdot 8$ & 83.1 & 81.7 & 0.04 \\
\hline $95 \% \mathrm{Cl}$ & $149 \cdot 0,152 \cdot 8$ & $146 \cdot 1,151 \cdot 0$ & $146 \cdot 7,151 \cdot 6$ & $146 \cdot 9,151 \cdot 8$ & $144 \cdot 9,149 \cdot 8$ & & $82 \cdot 5,84 \cdot 4$ & $81 \cdot 0,83 \cdot 7$ & $81 \cdot 4,84 \cdot 1$ & $81 \cdot 8,84 \cdot 4$ & $80 \cdot 4,83 \cdot 0$ & \\
\hline $\begin{array}{l}\text { Adjusted } \\
\text { model } \neq\end{array}$ & 0 & -2.8 & -2.5 & $-2 \cdot 2$ & $-4 \cdot 2$ & 0.01 & 0 & -1.5 & $-1 \cdot 1$ & -0.5 & -1.8 & 0.09 \\
\hline $95 \% \mathrm{Cl}$ & (ref) & $-5 \cdot 3,-0 \cdot 3$ & $-5 \cdot 1,0.03$ & $-4 \cdot 8,0 \cdot 4$ & $-6 \cdot 9,-1 \cdot 4$ & & (ref) & $-2 \cdot 8,-0 \cdot 2$ & $-2 \cdot 4,0 \cdot 3$ & $-1 \cdot 9,0 \cdot 8$ & $-3 \cdot 2,-0.4$ & \\
\hline
\end{tabular}

ref, Reference.

For details of subjects and procedures, see Methods.

Adjusted for age, sex, centre, BMI, physical activity, treatment with non-steroid anti-inflammatory drugs (yes/no), treatment with angiotensin converting enzyme inhibitors or angiotensin II receptor antagonists (yes/no), treatment with

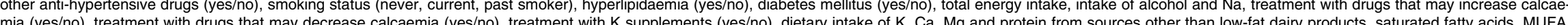
fibre, fruit and vegetable. All quantitative variables have been introduced in quintiles. $\ddagger$ With additional adjustment for intervention group. 
Table 3. Blood pressure (BP) levels and BP changes across quintiles of whole-fat dairy consumption*

(Means and $95 \% \mathrm{Cl}$ )

\begin{tabular}{|c|c|c|c|c|c|c|c|c|c|c|c|c|}
\hline \multirow[b]{2}{*}{ Sample size... } & \multicolumn{6}{|c|}{ Systolic BP } & \multicolumn{6}{|c|}{ Diastolic BP } \\
\hline & $\begin{array}{l}\text { Q1 } \\
458\end{array}$ & $\begin{array}{c}\text { Q2 } \\
458\end{array}$ & $\begin{array}{l}\text { Q3 } \\
458\end{array}$ & $\begin{array}{c}\text { Q4 } \\
458\end{array}$ & $\begin{array}{c}\text { Q5 } \\
458\end{array}$ & $P$ for trend & $\begin{array}{l}\text { Q1 } \\
458\end{array}$ & $\begin{array}{c}\text { Q2 } \\
458\end{array}$ & $\begin{array}{c}\text { Q3 } \\
458\end{array}$ & $\begin{array}{l}\text { Q4 } \\
458\end{array}$ & $\begin{array}{c}\text { Q5 } \\
458\end{array}$ & $P$ for trend \\
\hline \multicolumn{13}{|c|}{ Baseline, cross-sectional } \\
\hline Mean BP & 149.0 & $149 \cdot 1$ & $151 \cdot 7$ & $150 \cdot 5$ & $152 \cdot 4$ & 0.02 & $82 \cdot 0$ & 83.4 & $85 \cdot 2$ & 83.7 & 84.4 & 0.09 \\
\hline $95 \% \mathrm{Cl}$ & $147 \cdot 1,150 \cdot 8$ & $146 \cdot 5,151 \cdot 6$ & $149 \cdot 2,154 \cdot 2$ & $148 \cdot 0,153 \cdot 0$ & $149 \cdot 8,154 \cdot 9$ & & $81 \cdot 0,83 \cdot 0$ & $82 \cdot 0,84 \cdot 7$ & $83.8,86.5$ & $82 \cdot 3,85 \cdot 0$ & $83 \cdot 0,85 \cdot 8$ & \\
\hline Adjusted model $\dagger$ & 0 & -0.6 & 1.6 & 0.6 & $2 \cdot 4$ & 0.19 & 0 & 0.3 & 2.0 & 0.9 & $2 \cdot 3$ & 0.08 \\
\hline $95 \% \mathrm{Cl}$ & (ref) & $-3 \cdot 1,1 \cdot 9$ & $-1 \cdot 1,4 \cdot 4$ & $-2 \cdot 4,3.5$ & $-1 \cdot 0,5 \cdot 9$ & & (ref) & $-1 \cdot 0,1 \cdot 6$ & $0.5,3.4$ & $-0.6,2.5$ & $0.6,4 \cdot 1$ & \\
\hline \multicolumn{13}{|c|}{12 month cross-sectional } \\
\hline Mean BP & $147 \cdot 2$ & 148.5 & 149.8 & $150 \cdot 3$ & 149.5 & 0.28 & 81.5 & $82 \cdot 4$ & 83.9 & $83 \cdot 1$ & $82 \cdot 5$ & 0.87 \\
\hline $95 \% \mathrm{Cl}$ & $145 \cdot 5,148 \cdot 9$ & $146 \cdot 0,150 \cdot 9$ & $147 \cdot 3,152 \cdot 2$ & $147 \cdot 9,152 \cdot 8$ & $147 \cdot 0,151 \cdot 9$ & & $80 \cdot 6,82 \cdot 4$ & $81 \cdot 1,83.7$ & $82 \cdot 5,85 \cdot 2$ & $81 \cdot 8,84 \cdot 4$ & $81 \cdot 2,83 \cdot 9$ & \\
\hline Adjusted model¥ & 0 & 0.9 & $2 \cdot 3$ & 2.4 & 0.7 & 0.74 & & 1.0 & $2 \cdot 3$ & & & 0.35 \\
\hline $95 \% \mathrm{Cl}$ & (ref) & $-1.5,3.4$ & $-0.1,4 \cdot 7$ & $-0.1,4.9$ & $-1 \cdot 9,3 \cdot 3$ & & (ref) & $-0 \cdot 2,2 \cdot 3$ & $1 \cdot 0,3 \cdot 5$ & $0.4,3 \cdot 0$ & $0.3,2 \cdot 9$ & \\
\hline \multicolumn{13}{|c|}{ Longitudinal, baseline exposure 12 months BP } \\
\hline Mean BP & $148 \cdot 2$ & $147 \cdot 6$ & $149 \cdot 4$ & 148.5 & $151 \cdot 6$ & 0.002 & 81.9 & 82.5 & $83 \cdot 3$ & $82 \cdot 7$ & 83.1 & 0.34 \\
\hline $95 \% \mathrm{Cl}$ & $146 \cdot 5,149 \cdot 9$ & $145 \cdot 1,150 \cdot 0$ & $146 \cdot 9,151 \cdot 8$ & $146 \cdot 1,151 \cdot 0$ & $149 \cdot 1,154 \cdot 1$ & & $81 \cdot 0,82 \cdot 8$ & $81 \cdot 2,83 \cdot 8$ & $82 \cdot 0,84 \cdot 6$ & $81 \cdot 4,84.0$ & $81 \cdot 7,84.4$ & \\
\hline Adjusted model & 0 & -0.8 & 0.2 & $-1 \cdot 2$ & 0.0 & 0.84 & & -0.3 & 0.2 & -0.2 & 0.3 & 0.61 \\
\hline $95 \% \mathrm{Cl}$ & (ref) & $-3 \cdot 3,1 \cdot 7$ & $-2.5,2.9$ & $-4 \cdot 1,1 \cdot 7$ & $-3.4,3.3$ & & (ref) & $-1 \cdot 6,0.9$ & $-1 \cdot 3,1 \cdot 5$ & $-1 \cdot 6,1 \cdot 3$ & $-1 \cdot 4,2 \cdot 0$ & \\
\hline
\end{tabular}

ref, Reference.

For details of subjects and procedures, see Methods.

† Adjusted for age, sex, centre, BMI, physical activity, treatment with non-steroid anti-inflammatory drugs (yes/no), treatment with angiotensin converting enzyme inhibitors or angiotensin II receptor antagonists (yes/no), treatment with other anti-hypertensive drugs (yes/no), smoking status (never, current, past smoker), hyperlipidaemia (yes/no), diabetes mellitus (yes/no), total energy intake, intake of alcohol and Na, treatment with drugs that may increase calcaesour (y) , $\neq$ With additional adjustment for intervention group. 
analysis after 12 months follow-up). However, the point estimates for the difference in DBP between the upper and lower quintiles of low-fat dairy product intake always had a negative sign and were statistically significant, suggesting a modest non-significant inverse association. Neither the comparisons across extreme quintiles nor the linear trend tests in the longitudinal analysis with change in BP as outcome or in the dynamic longitudinal analysis were statistically significant (data not shown).

No significant trends were observed in the analyses using whole-fat dairy product consumption as exposure. It is noteworthy that, contrary to what we observed for low-fat dairy products, the point estimates for the adjusted comparisons between extreme quintiles of whole-fat dairy product consumption had always but once a positive sign, suggesting a modest non-significant positive association. In the longitudinal analysis with change in BP as outcome and in the dynamic longitudinal analysis no comparison among extreme quintiles was statistically significant (data not shown).

When we additionally adjusted for fish or whole grain intake, the results did not substantially change (data not shown).

We found no interaction between low-fat and whole-fat dairy product consumption and obesity, diabetes mellitus, hypertension or treatment with anti-hypertensive agents (data not shown).

\section{Discussion}

The results of the present study suggest a beneficial effect of low-fat dairy product consumption on BP.

To our knowledge, the present study is the first large prospective cohort observing an inverse association between low-fat dairy products and BP in an older population at high cardiovascular risk during a 12-month follow-up. The large sample size and the thorough information collected allowed us to comprehensively adjust for potential confounding, including both dietary and non-dietary confounders. Nonetheless, the current study has some limitations. First, we acknowledge that our participants are not a representative sample of the general population in the statistical sense. This is true for most samples used in randomized trials and epidemiological cohorts; however, this does not preclude performing scientific inferences ${ }^{(22)}$. In addition to this, the observed magnitude of the BP effect associated with low-fat dairy product intake was not very large, i.e. in the longitudinal analysis there were reductions of up to $4 \mathrm{mmHg}$ in SBP. Nevertheless, reductions of $2-5 \mathrm{mmHg}$ in average $\mathrm{BP}$ levels at the population level may reduce all-cause mortality, fatal CHD and fatal stroke by $7 \%, 9 \%$ and $14 \%$, respectively ${ }^{(3)}$. This is important from a public health perspective. Another potential limitation is that residual confounding might explain part of the observed associations because dietary variables are imperfectly measured by the instruments used in nutritional epidemiology ${ }^{(23)}$. Furthermore, it could be argued that the observed lower BP levels among consumers of low-fat dairy products may not be attributable to a specific effect of these foods but to other aspects of an overall healthier lifestyle that are not captured by the information collected in Table 1 . This is unlikely, however, because subjects in the top quintile of low-fat dairy product intake had a higher BMI, a lower educational level and alcohol intake and were less physically active and less likely to be past smokers than those in the lowest quintile. More importantly, if the parameters that we estimated for the association between dairy product consumption and BP had been substantially reduced when adjusting for potential confounders, we could conclude that the observed effect might still be attributable to unmeasured confounding. This was not the case, since multivariate-adjusted estimates taking into account potential lifestyle-related confounders did not show a noticeable reduction in the magnitude of the effect with regard to unadjusted estimates. Therefore, it is unlikely that a large degree of residual confounding might be responsible for the observed association. Moreover, some of the observed associations were not linear. Indeed, a linear, monotonically decreasing pattern would have strengthened the case for a causal relationship. We do not have a definitive explanation for the non-linear shape, although random error and non-differential misclassification of exposure for intermediate quintiles may contribute to this shape of the dose-response curve. Despite this, it is noteworthy that most adjusted point estimates for the fifth quintile showed the lowest value for BP when low-fat dairy products were considered as the exposure.

Findings from other studies also support the results from the present study. First, in some cohorts a beneficial effect of total dairy consumption on BP was observed ${ }^{(6,24,25)}$. In the Coronary Artery Risk Development in Young Adults cohort study ${ }^{(6)}$, the 10-year cumulative incidence of elevated BP was inversely associated with total dairy intake only among overweight participants. In addition, the protective effect of an increment of one daily eating occasion of a reduced-fat dairy product was higher than the protective effect of that of a serving of a high-fat dairy product. These findings also suggested a specific protective effect of low-fat dairy products and provided consistency for the present results. However, after 15-years of follow-up in the same cohort $^{(26)}$, only milk and dairy desserts, but not total dairy products, were inversely related to elevated BP. In a crosssectional study conducted in Greece ${ }^{(25)}$, an inverse association between dairy product consumption and DBP was also found; however, there was no significant association for SBP.

Other studies have suggested a differential effect on BP of low- $v$. whole-fat dairy consumption, with a beneficial effect shown for low-fat products ${ }^{(5,7)}$. In a Spanish cohort study including middle-aged adults (the SUN Study) $)^{(5)}$, an inverse association between low-fat dairy products, but not wholefat dairy products, and the risk of hypertension was found. Consistently, total dairy product intake and low-fat dairy intake have been found to be negatively associated with the risk of hypertension among middle-aged and older women in a recent prospective cohort study ${ }^{(27)}$. In another study conducted in an elderly population ${ }^{(28)}$, only total dairy product intake was modestly associated with lower levels of DBP; however, no statistical differences were found when separating low-fat dairy products from whole-fat dairy products. The DASH trial ${ }^{(4)}$ and others ${ }^{(29)}$ have shown beneficial effects on BP of dietary patterns including low-fat dairy products; however, the intervention was not focused exclusively on these foods. No other randomized trial has assessed the isolated effect on BP of low-fat dairy products. 
The differential association between low-fat dairy product consumption and whole-fat dairy product consumption and BP found in the present cohort is consistent with the results of other studies ${ }^{(5,7,27)}$ and might be explained by the deleterious effect of the SFA contained in whole-fat dairy products on endothelial function ${ }^{(11)}$.

Nonetheless, we found no statistically significant associations when assessing how BP changed with changes in the exposure to low-fat dairy products, and this finding should temper our conclusions. However, point estimates for differences between extreme quintiles were in the expected direction. The lack of statistical significance for some analyses could be explained by the advanced age of our participants and their high frequency and long duration of hypertension, making it difficult to attain substantial changes in BP with only a change in a single nutritional exposure during a 12-month period.

In the CARDIA cohort study ${ }^{(6)}$, there was a significant interaction between a higher BMI and the consumption of dairy products on the risk of high BP. Since participants of the PREDIMED trial are at high cardiovascular risk, only very few of them had a normal weight. Therefore, our statistical power to perform subgroup analyses regarding overweight status was insufficient. However, we found no interaction between low-fat or whole-fat dairy consumption and obesity for the association with BP levels.

In conclusion, low-fat dairy products could play an important role in the prevention of hypertension and may be helpful in the control and reduction of BP levels. The current study fills one gap in the available evidence about the effects of dairy products in elderly high-risk populations, suggesting that low-fat dairy products could also be potentially beneficial in this group. To confirm our findings, large clinical trials designed specifically to assess the role of low-fat dairy products on BP levels or on the incidence of hypertension should be conducted.

\section{Acknowledgements}

The authors thank the participants in the PREDIMED study for their continued collaboration, Ana Sánchez-Tainta for her technical support and Dr Álvaro Alonso for his suggestions and advice. The PREDIMED study is funded by public resources from the Spanish Ministry of Health (Fondo de Investigación Sanitaria PI04233, PI051839, PI070240, PI052368, PI052584, PI051458, PI042234, RTIC G03/140, RD06/0045/0000 and CIBER CB06/03 Fisiopatología de la Obesidad y Nutrición) and from the Generalitat Valenciana (GRUPOS2004-43 and ACOMP06109). The authors declare no conflict of interest.

\section{References}

1. Chobanian AV, Bakris GL, Black HR, et al. (2003) The Seventh Report of the Joint National Committee on Prevention, Detection, Evaluation, and Treatment of High Blood Pressure. The JNC 7 Report. JAMA 289, 2560-2572.

2. Lopez AD, Mathers CD, Ezzati M, Jamison DT \& Murray CJL (2006) Global and regional burden of disease and risk factors, 2001: systematic analysis of population health data. Lancet 367, 1747-1757.

3. Appel LJ, Brands MW, Daniels SR, Karanja N, Elmer PJ \& Sacks FM (2006) Dietary approaches to prevent and treat hypertension. A scientific statement from the American Heart Association. Hypertension 47, 296-308.

4. Appel LJ, Moore TJ, Obarzanek E, et al. (1997) A clinical trial of the effects of dietary patterns on blood pressure. $N$ Engl $J$ Med 336, $1117-1124$.

5. Alonso A, Beunza JJ, Delgado-Rodríguez M, Martínez JA \& Martínez-González MA (2005) Low-fat dairy consumption and reduced risk of hypertension: the Seguimiento Universidad de Navarra (SUN) cohort. Am J Clin Nutr 82, 972-979.

6. Pereira MA, Jacobs DR, Van Horn L, Slattery ML, Kartashov AI \& Ludwig DS (2002) Dairy consumption, obesity, and the insulin resistance syndrome in Young Adults. The CARDIA Study. JAMA 287, 2081-2089.

7. Djoussé L, Pankow JS, Hunt SC, Heiss G, Province MA, Kabagambe EK \& Ellison RC (2006) Influence of saturated fat and linolenic acid on the association between intake of dairy products and blood pressure. Hypertension 48, 335-341.

8. Sutoo D \& Akiyama K (1997) Regulation of blood pressure with calcium-dependent dopamine synthesizing system in the brain and its related phenomena. Brain Res Rev 25, 1-26.

9. Vaskonen T (2003) Dietary minerals and modification of cardiovascular risk factors. J Nutr Biochem 14, 492-506.

10. Jauhiainen T \& Korpela R (2007) Milk peptides and blood pressure. J Nutr 137, 825S-829S.

11. Keogh JB, Grieger JA, Noakes M \& Clifton PM (2005) Flowmediated dilatation is impaired by a high-saturated fat diet but not by a high carbohydrate diet. Arterioscler Thromb Vasc Biol 25, 1274-1279.

12. Estruch R, Martinez-Gonzalez MA, Corella D, et al. (2006) Effects of a Mediterranean-style diet on cardiovascular risk factors: a randomized trial. Ann Intern Med 145, 1-11.

13. Willett WC (1998) Nutritional Epidemiology, 2nd ed. New York, NY: Oxford University Press.

14. World Medical Association (2004) World Medical Association Declaration of Helsinki. Tokyo: World Medical Association.

15. Martin-Moreno JM, Boyle P, Gorgojo L, Maisonneuve P, Fernandez-Rodriguez JC, Salvini S \& Willett WC (1993) Development and validation of a food frequency questionnaire in Spain. Int J Epidemiol 22, 512-519.

16. Moreiras O, Carvajal A \& Cabrera L (2005) Tablas de composición de alimentos (Food Composition Tables), 9th ed. Madrid: Pirámide.

17. Mataix Verdú J \& Mañas Almendros M (2003) Tabla de composición de alimentos (Food Composition Tables), 4th ed. Granada: Universidad de Granada.

18. O'Brien E, Mee F, Atkins N \& Thomas M (1996) Evaluation of three devices for self-measurement of blood pressure according to the revised British Hypertension Society Protocol: the Omron HEM-705CP, Phillips HP5332, and Nissei DS-175. Blood Pressure Monitoring 1, 55-61.

19. Iglesias Bonilla P, Mayoral Sánchez E, Lapetra Peralta J, Iborra Oquendo M, Villalba Alcalá F \& Cayuela Domínguez A (2002) Validación de dos sistemas de automedida de presión arterial, modelos OMRON HEM-705 CP y OMRON M1 (HEM 422C2-E). Atencion Primaria (Primary Health Care) 30, $22-28$.

20. Elosua R, Garcia M, Aguilar A, Molina L, Covas MI \& Marrugat J (2000) Validation of the Minnesota Leisure Time Physical Activity Questionnaire in Spanish Women. Investigators of the MARATHOM Group. Med Sci Sports Exerc 32, $1431-1437$.

21. Elosua R, Marrugat J, Molina L, Pons S \& Pujol E (1994) Validation of the Minnesota Leisure Time Physical Activity Questionnaire in Spanish men. The MARATHOM Investigators. Am J Epidemiol 139, 1197-1209. 
22. Rothman KJ (2002) Epidemiology: An Introduction. New York, NY: Oxford University Press.

23. Day NE, Wong MY, Bingham $\mathrm{S}$, Khaw KT, Luben $\mathrm{R}$, Michels KB, Welch A \& Wareham NJ (2004) Correlated measurement error-implications for nutritional epidemiology. Int J Epidemiol 33, 1373-1381.

24. Moore LL, Singer MR, Bradlee ML, Djoussé L, Proctor MH, Cupples LA \& Ellison RC (2005) Intake of fruits, vegetables, and dairy products in early childhood and subsequent blood pressure change. Epidemiology 16, 4-11.

25. Psaltopoulou T, Naska A, Orfanos P, Trichopoulos D, Mounyokalakis T \& Trichopoulou A (2004) Olive oil, the Mediterranean diet, and arterial blood pressure: the Greek European Prospective Investigation into Cancer and Nutrition (EPIC) study. Am J Clin Nutr 80, 1012-1018.

26. Steffen LM, Kroenke CH, Yu X, Pereira MA, Slattery ML, Van Horn L, Gross MD \& Jacobs DR (2005) Associations of plant food, dairy product, and meat intakes with 15 -y incidence of elevated blood pressure in young black and white adults: the Coronary Artery Risk Development in Young Adults (CARDIA) Study. Am J Clin Nutr 82, 1169-1177.

27. Wang L, Manson JE, Buring JE, Lee IM \& Sesso HD (2008) Dietary intake of dairy products, calcium, and vitamin D and the risk of hypertension in middle-aged and older women. Hypertension 51, 1-7.

28. Snijder MB, van der Heijden AA, van Dam RM, Stehouwer CD, Hiddink GJ, Nijpels G, Heine RJ, Bouter LM \& Dekker JM (2007) Is higher dairy consumption associated with lower body weight and fewer metabolic disturbances? The Hoorn Study. Am J Clin Nutr 85, 989-995.

29. Nowson CA, Worsley A, Margerison C, Jorna MK, Godfrey SJ \& Booth A (2005) Blood pressure change with weight loss is affected by diet type in men. Am J Clin Nutr 81, 983-989. 